\title{
The Solvability of the Initial-Boundary Value Problems for a Nonlinear Schrödinger Equation with a Special Gradient Term
}

\author{
G. Yagub, N.S. Ibrahimov, and M. Zengin
}

In this paper, the initial-boundary value problems for the two-dimensional nonlinear Schrödinger equation with a special gradient term with purely imaginary coefficients in the nonlinear part, when the coefficients of the equation are measurable bounded functions, are considered. The existence and uniqueness of solutions of the first and second initial-boundary value problems is proved almost everywhere.

Key words: Schrödinger equation, special gradient term, existence and uniqueness, first and second initial-boundary value problems.

Mathematical Subject Classification 2010: 35D, 35M, 35Q.

\section{Introduction}

In this paper we study the correct formulation of the initial-boundary value problems for the nonlinear Schrödinger equation with a special gradient term. As it is well known, the Schrödinger equation with a special gradient term and the initial-boundary value problems for this equation appear in quantum mechanics, nuclear physics, nonlinear optics and other fields of modern physics and engineering $[3,15,19]$. Especially in quantum mechanics and nonlinear optics in the study of the motion of charged particles in a nonhomogeneous environment, the Schrödinger equation has a special gradient term. Therefore, the study of boundary value problems of this type of Schrödinger equations is of interest both for theoretical and practical problems. It should be noted that the initial-boundary value problems for the linear and nonlinear Schrödinger equations in various formulations were previously studied in detail in $[1,2,4-8,16,18]$. However, even for the linear Schrödinger equation with a special gradient term, initial-boundary value problems are poorly investigated $[10,17]$. In this paper, we study the questions of the existence and uniqueness of solutions of boundary value problems for the linear one-dimensional and two-dimensional Schrödinger equations with a special gradient term, where the coefficients are square integrable functions. Note that the initial-boundary value problem for the nonlinear Schrödinger equation with a special gradient term has not been studied yet. Therefore, the study of the

(c) G. Yagub, N.S. Ibrahimov, and M. Zengin, 2018 
existence and uniqueness of the initial-boundary value problems for the nonlinear Schrödinger equation with a special gradient term is of scientific and practical interest.

\section{The existence and uniqueness of a solution of the first initial-boundary value problem}

In this paper, we will first examine the first initial-boundary value problem for the nonlinear Schrödinger equation with a special gradient term with purely imaginary coefficients in the nonlinear part in the case when the coefficients of the equation are bounded measurable functions.

Let $D$ be a bounded convex domain in $R^{2}$, with the boundary $\Gamma$, that is assumed to be smooth enough; $x=\left(x_{1}, x_{2}\right)$ be an arbitrary point of the domain $D ; T>0$ be a given number; $0 \leq t \leq T ; \Omega_{t}=D \times(0, t) ; \Omega=\Omega_{T} ; S=\Gamma \times(0, T)$ be the lateral surface of $\Omega ; C^{k}([0, T], B)$ be a Banach space of the functions, $k$-times differentiable in the interval $[0, T]$ with values in the Banach space $B ; L_{p}(D)$ be a Lebesque space of the functions summerable over the module with an order $p \geq 1$ of functions for which the $p$-th power of the absolute value is summable; $L_{2}(0, T ; B)$ be a Banach space of the functions defined and square-summable on the interval $[0, T]$ with values from the Banach space $B ; L_{\infty}(0, T ; B)$ be a Banach space of measurable bounded functions on $(0, T)$ with values from the Banach space $B$. The Sobolev spaces $W_{p}^{k}(0, l), W_{p}^{k, m}(\Omega), p \geq 1, k \geq 0, m \geq 0$ are defined as in $[11,13]$.

Consider the initial-boundary problem of determination of a function $\psi=$ $\psi(x, t)$ in the domain $\Omega$ from the conditions

$$
\begin{aligned}
i \frac{\partial \psi}{\partial t}+a_{0} \Delta \psi & +i a_{1}(x) \nabla \psi \\
& -a(x) \psi+v(x) \psi+i a_{2}|\psi|^{2} \psi=f(x, t), \quad(x, t) \in \Omega, \\
\psi(x, 0) & =\varphi(x), \quad x \in D,\left.\psi\right|_{S}=0
\end{aligned}
$$

where $i=\sqrt{-1} ; a_{0}>0, a_{2}>0$ are given numbers; $\Delta=\frac{\partial^{2}}{\partial x_{1}^{2}}+\frac{\partial^{2}}{\partial x_{2}^{2}}$ is the Laplace operator; $\nabla=\left(\frac{\partial}{\partial x_{1}}, \frac{\partial}{\partial x_{2}}\right) ; a(x), v(x)$ are measurable bounded functions satisfying the conditions

$$
\begin{aligned}
& 0 \leq a(x) \leq \mu_{0}, \quad x \in D, \quad \quad \mu_{0}=\text { const }>0 ; \\
& |v(x)| \leq b_{0}, \quad x \in D, \quad b_{0}=\text { const }>0 ;
\end{aligned}
$$

$a_{1}(x)=\left(a_{11}(x), a_{12}(x)\right)$ is a given vector-function with the components satisfying the conditions

$$
\left|a_{1 j}(x)\right| \leq \mu_{1},\left|\frac{\partial a_{1 j}(x)}{\partial x_{k}}\right| \leq \mu_{2}, \quad x \in D, j, k=1,2, \mu_{1}, \mu_{2}=\text { const }>0 ;
$$

$\phi(x), f(x, t)$ are complex valued functions satisfying the conditions

$$
\varphi \in \stackrel{\circ}{W}_{2}^{2}(D), \quad f \in W_{2}^{0,1}(\Omega) .
$$


It is clear that the problem of determination of $\psi=\psi(x, t)$ from conditions (2.1), (2.2) is an initial-boundary problem for the two-dimensional nonlinear Schrödinger equation of the form (2.1).

Definition 2.1. The function $\psi=\psi(x, t)$ from the space

$$
B_{0} \equiv C^{0}\left([0, T], \stackrel{\circ}{W}_{2}^{2}(D)\right) \bigcap C^{1}\left([0, T], L_{2}(D)\right)
$$

is called a generalized solution to (2.1), (2.2) if it satisfies equation (2.1) for almost all $x \in D$ and for any $t \in[0, T]$, and initial and boundary conditions (2.2) for almost all $x \in D$ and almost all $(\xi, t) \in S$, correspondingly.

Theorem 2.2. Let the functions $a(x), v(x), a_{1}(x), \varphi(x), f(x, t)$ satisfy conditions (2.3)-(2.6). Then the initial-boundary problem (2.1), (2.2) has a unique solution in the space $B_{0}$, and for this solution the estimate

$$
\begin{aligned}
\|\psi(\cdot, t)\|_{\stackrel{\circ}{2}_{2}^{2}(D)} & +\left\|\frac{\partial \psi(\cdot, t)}{\partial t}\right\|_{L_{2}(D)} \\
& \leq c_{0}\left(\|\phi\|_{\stackrel{\circ}{2}_{2}^{2}(D)}+\|f\|_{W_{2}^{0,1}(\Omega)}+\|\phi\|_{W_{2}^{2}(D)}^{3}\right), \quad t \in[0, T],
\end{aligned}
$$

is valid, where the constant $c_{0}>0$ does not depend on $\phi, f$ and $t$.

Proof. We choose the fundamental in $\stackrel{\circ}{W}_{2}^{2}(D)$ and orthonormal in $L_{2}(D)$ system of functions $u_{k}=u_{k}(x), k=1,2, \ldots$, for example, the system of eigenfunctions of the following spectral problem:

$$
L X(x)=\lambda X(z), \quad x \in D,\left.X\right|_{\Gamma}=0
$$

at $\lambda=\lambda_{k}, k=1,2, \ldots$, where the operator $L$ is defined by the formula

$$
L=-a_{0} \Delta+a(x)
$$

with Dirichlet boundary conditions.

Notice that (2.9) is a spectral problem for the two-dimensional equation of elliptic type studied in [15]. Therefore, by the help of the result obtained in this paper, we can state that the spectral problem (2.10) has nontrivial solutions $X=$ $u_{k}(x), k=1,2, \ldots$, at $\lambda=\lambda_{k}, k=1,2, \ldots$, forming a spectra of the problem, and these solutions form a basis in the spaces $\stackrel{\circ}{W}{ }_{2}^{1}(D), \stackrel{\circ}{W}{ }_{2}^{2}(D)$. The conditions of orthonormality in $L_{2}(D)$ and orthogonality in $\stackrel{\circ}{W}_{2}^{1}(D), W(D)$ are given in the form

$$
\left(u_{k}, u_{m}\right)_{L_{2}(D)}=\int_{D} u_{k}(x) u_{m}(x) d x=\delta_{k}^{m}
$$

where $\delta_{k}^{m}$ are Kronecker symbols

$$
\delta_{k}^{m}=\left\{\begin{array}{ll}
1 & k=m, \\
0, & k \neq m
\end{array}, \quad k, m=1,2, \ldots\right.
$$


It is clear that the functions $u_{k}(x), k=1,2, \ldots$ are orthogonal in the following sense:

$$
\begin{aligned}
& {\left[u_{k}, u_{m}\right]=\left(u_{k}, u_{m}\right)_{\stackrel{\circ}{2}_{2}^{1}(D)}=\left(L u_{k}, u_{m}\right)_{L_{2}(D)}} \\
& =e \int_{D}\left(a_{0} \sum_{j}^{2} \frac{\partial u_{k}}{\partial x_{j}} \frac{\partial u_{m}}{\partial x_{j}}+a(x) u_{k} u_{m}\right) d x \\
& =\lambda_{k} \delta_{k}^{m}, \quad k, m=1,2, \ldots ; \\
& \left\{u_{k}, u_{m}\right\}=\left(L u_{k}, L u_{m}\right)_{L_{2}(D)}=\left(u_{k}, u_{m}\right)_{W_{2}^{2}(D)}=\lambda_{k} \delta_{k}^{m}, \quad k, m=1,2, \ldots
\end{aligned}
$$

Due to the assumption $a(x) \geq 0$, all eigenvalues $\lambda=\lambda_{k}, k=1,2, \ldots$ are real, positive and numbered in the increasing order

$$
0 \leq \lambda_{1} \leq \lambda_{2} \leq \lambda_{3} \leq \cdots \leq \lambda_{k} \leq \cdots, \quad \lambda_{k} \rightarrow \infty \quad \text { as } k \rightarrow \infty
$$

We additionally assume that

$$
\left\|u_{k}\right\|_{W_{2}^{2}(D)} \leq \tilde{d}_{k}<+\infty, \quad k=1,2, \ldots
$$

where $\tilde{d}_{k}, k=1,2, \ldots$, are positive constants.

By Galerkin's method, we seek the approximate solution in the form

$$
\psi^{N}(x, t)=\sum_{k=1}^{N} c_{k}^{N}(t) u_{k}(x),
$$

where $c_{k}^{N}(t)=\left(\psi^{N}(\cdot, t), u_{k}\right)_{L_{2}(\partial)}, k=1,2, \ldots, N$, are defined by the conditions

$$
\begin{aligned}
& i \frac{d}{d t}\left(\psi^{N}(\cdot, t), u_{k}\right)_{L_{2}(D)}-\left(L \psi^{N}(\cdot, t), u_{k}\right)_{L_{2}(D)} \\
& \quad+i\left(a_{1}(\cdot) \nabla \psi^{N}(\cdot, t), u_{k}\right)_{L_{2}(D)}+\left(v(\cdot) \psi^{N}(\cdot, t), u_{k}\right)_{L_{2}(D)} \\
& \quad+i\left(a_{2}\left|\psi^{N}\right|^{2} \psi^{N}, u_{k}\right)=f_{k}(t), \quad k=1,2, \ldots, N, t \in[0, T], \\
& c_{k}^{N}(0)=\left(\psi^{N}(\cdot, 0), u_{k}\right)_{L_{2}(D)}=\varphi_{k}, \quad k=1,2, \ldots, N .
\end{aligned}
$$

Here $f_{k}(t)=\left(f(\cdot, t), u_{k}\right)_{L_{2}(D)}, \phi_{k}=\left(\phi, u_{k}\right)_{L_{2}(D)}, k=1,2, \ldots, N$. System $(2.18)$ consists of the system of $N$ nonlinear ordinary differential equations. It follows from assumptions (2.3)-(2.6) and the properties of $u_{k}(x), k=1,2, \ldots$ that the second, third, fourth and fifth terms in the left- and the right-hand sides are continuous on each set $\left\{t \in[0, T],\left|c_{k}^{N}\right| \leq\right.$ const $\}$ of the functions $t, c_{k}^{N}, k=$ $1,2, \ldots, N$. Therefore, for the existence of a solution to the Cauchy problem, it is sufficient to show that the solutions are bounded uniformly with respect to $t \in$ $[0, T]$ for any $T>0$ (see $[9,12-14])$. To establish the boundedness, we have to prove the following. 
Lemma 2.3. For the solutions of problem (2.18), (2.19), the estimate

$$
\begin{array}{rl}
\sum_{k=1}^{N}\left|c_{k}^{N}(t)\right|^{2}+\sum_{k=1}^{N}\left|\frac{d c_{k}^{N}(t)}{d t}\right|^{2} & \leq\left\|\psi^{N}(\cdot, t)\right\|_{W_{2}^{2}(D)}+\left\|\frac{\partial \psi^{N}(\cdot, t)}{\partial t}\right\|_{L_{2}(D)}^{2} \\
& \leq c_{1}\left(\|\phi\|_{W_{2}^{2}(D)}^{2}+\|f\|_{W_{2}^{0,1}(\Omega)}^{2}+\|\phi\|_{W_{2}^{1}(D)}^{6}\right) \\
t & t \in[0, T], N=1,2, \ldots
\end{array}
$$

is valid.

Proof. Multiplying each $k$-th equation from (2.18) by $\bar{c}_{k}^{N}(t)$, summing the obtained equalities over $k$ from 1 to $N$, integrating over $t$ from zero to $t \leq T$ and then using the formula of integration by parts and the condition $\left.u_{k}\right|_{\Gamma}=0, k=$ $1,2, \ldots$, we get

$$
\begin{aligned}
\int_{\Omega_{t}}\left(i \frac{\partial \psi^{N}}{\partial t} \bar{\psi}^{N}\right. & -a_{0}(x)\left|\nabla \psi^{N}\right|^{2}+i a_{1}(x) \nabla \psi^{N} \bar{\psi}^{N} \\
& \left.-a(x)\left|\psi^{N}\right|^{2}+v(x)\left|\psi^{N}\right|^{2}+i a_{2}\left|\psi^{N}\right|^{4}\right) d x d \tau \\
& =2 i \int_{\Omega_{t}} \operatorname{Im}\left(f \bar{\psi}^{N}\right) d x d \tau, \quad t \in[0, T]
\end{aligned}
$$

Subtracting from this equality its complex conjugate, we get the validity of the equality

$$
\begin{aligned}
i \int_{\Omega_{t}}\left(\frac{\partial \psi^{N}}{\partial t} \bar{\psi}^{N}\right. & \left.+\frac{\partial \bar{\psi}^{N}}{\partial t} \psi^{N}\right) d x d \tau \\
& +i \int_{\Omega_{t}}\left(a_{1}(x) \nabla \psi^{N} \bar{\psi}^{N}+a_{1}(x) \nabla \bar{\psi}^{N} \psi^{N}\right) d x d \tau \\
& +2 i a_{2} \int_{\Omega_{t}}\left|\psi^{N}\right|^{4} d x d \tau=2 i \int_{\Omega_{t}} \operatorname{Im}\left(f \bar{\psi}^{N}\right) d x d \tau, \quad t \in[0, T]
\end{aligned}
$$

Using differentiability of the functions $a_{1 j}(x), j=1,2$, the last one may be written as

$$
\begin{gathered}
\int_{\Omega_{t}} \frac{\partial}{\partial t}\left|\psi^{N}\right|^{2} d x d \tau+\int_{\Omega_{t}} \sum_{j=1}^{2} \frac{\partial}{\partial x_{j}}\left(a_{1 j}(x)\left|\psi^{N}\right|^{2}\right) d x d \tau+2 a_{2} \int_{\Omega_{t}}\left|\psi^{N}\right|^{4} d x d \tau \\
=\int_{\Omega_{t}} \sum_{j=1}^{2} \frac{\partial a_{1 j}(x)}{\partial x_{j}}\left|\psi^{N}\right|^{2} d x d \tau+2 \int_{\Omega_{t}} \operatorname{Im}\left(f \bar{\psi}^{N}\right) d x d \tau, \quad t \in[0, T] .
\end{gathered}
$$

Considering that the functions $u_{k}=u_{k}(x), k=1,2, \ldots$ satisfy the homogeneous boundary conditions $\left.u_{k}\right|_{\Gamma}=0, k=1,2, \ldots$, from decomposition (2.17) we have

$$
\left.\psi^{N}(x, t)\right|_{\Gamma}=0, \quad t \in(0, T), N=1,2, \ldots
$$


Taking into account the second terms and the conditions imposed on the coefficients of the equation from equality (2.21), one can easily obtain the validity of the inequality

$$
\begin{aligned}
& \left\|\psi^{N}(\cdot, t)\right\|_{L_{2}(D)}^{2}+2 a_{2} \int_{\Omega_{t}}\left|\psi^{N}\right|^{4} d x d \tau \\
& \quad \leq\left\|\psi^{N}(\cdot, 0)\right\|_{L_{2}(D)}^{2}+\|f\|_{L_{2}(\Omega)}^{2}+\left(2 \mu_{2}+1\right) \int_{0}^{t}\left\|\psi^{N}(\cdot, \tau)\right\|_{L_{2}(D)}^{2} d \tau
\end{aligned}
$$

for all $t \in[0, T]$. Using formula (2.17), we can write the relation

$$
\left\|\psi^{N}(\cdot, 0)\right\|_{L_{2}(D)}^{2}=\sum_{k=1}^{N}\left|c_{k}^{N}(0)\right|^{2} \leq \sum_{k=1}^{\infty}\left|\varphi_{k}\right|^{2}=\|\varphi\|_{L_{2}(D)}^{2} .
$$

With the help of this relation, from (2.23), we get

$$
\begin{aligned}
& \left\|\psi^{N}(\cdot, t)\right\|_{L_{2}(D)}^{2}+2 a_{2} \int_{\Omega_{t}}\left|\psi^{N}\right|^{4} d x d \tau \\
& \quad \leq\|\phi\|_{L_{2}(D)}^{2}+\|f\|_{L_{2}(\Omega)}^{2}+\left(2 \mu_{2}+1\right) \int_{0}^{t}\left\|\psi^{N}(\cdot, \tau)\right\|_{L_{2}(D)}^{2} d \tau, \quad t \in[0, T] .
\end{aligned}
$$

Using this inequality and Gronwall's lemma, it is not difficult to get the estimate

$$
\begin{aligned}
\left\|\psi^{N}(\cdot, t)\right\|_{L_{2}(D)}^{2} & +2 a_{2} \int_{\Omega_{z}}\left|\psi^{N}\right|^{4} d x d \tau \\
& \leq c_{2}\left(\|\phi\|_{L_{2}(D)}^{2}+\|f\|_{L_{2}(\Omega)}^{2}\right), \quad t \in[0, T] .
\end{aligned}
$$

Now we estimate $\frac{\partial \psi^{N}}{\partial t}$. For this purpose, we write system (2.18) in the form

$$
\begin{aligned}
i \frac{d}{d t}\left(\psi^{N}\right. & \left.(\cdot, t), u_{k}\right)_{L_{2}(D)}-\left(a_{0} \nabla \psi^{N}(\cdot, t), \nabla u_{k}\right)_{L_{2}(D)}-\left(a(\cdot) \psi^{N}(\cdot, t), u_{k}\right)_{L_{2}(D)} \\
& +\left(v(\cdot) \psi^{N}(\cdot, t), u_{k}\right)_{L_{2}(D)}+i\left(a_{1}(\cdot) \nabla \psi^{N}(\cdot, t), u_{k}\right)_{L_{2}(D)} \\
& +\left(i a_{2}\left|\psi^{N}(\cdot, t)\right|^{2} \psi^{N}(\cdot, t), u_{k}\right)_{L_{2}(D)}=f_{k}(t), \quad k=1,2, \ldots, N .
\end{aligned}
$$

We differentiate both sides of this system with respect to $t$ and multiply the $k$-th equation of the obtained system by $\frac{d \bar{c}_{k}^{N}(t)}{d t}$, and then sum the obtained equations over $k$ from 1 to $N$. Then, integrating the obtained equality on the interval $(0, t)$, we have

$$
\begin{aligned}
& \int_{\Omega_{t}}\left(i \frac{\partial^{2} \psi^{N}}{\partial t^{2}} \frac{\partial \bar{\psi}^{N}}{\partial t}-a_{0} \sum_{j=1}^{2}\left|\frac{\partial^{2} \psi^{N}}{\partial t \partial x_{j}}\right|^{2}+i \sum_{j=1}^{2} a_{1 j}(x) \frac{\partial^{2} \psi^{N}}{\partial x_{j} \partial t} \frac{\partial \bar{\psi}^{N}}{\partial t}\right. \\
& \left.-a(x)\left|\frac{\partial \psi^{N}}{\partial t}\right|^{2}+v(x)\left|\frac{\partial \psi^{N}}{\partial t}\right|^{2}\right) d x d \tau
\end{aligned}
$$




$$
\begin{aligned}
& +i a_{2} \int_{\Omega_{t}} \frac{\partial}{\partial t}\left(\left|\psi^{N}\right|^{2} \psi^{N}\right) \frac{\partial \bar{\psi}^{N}}{\partial t} d x d \tau \\
& =\int_{\Omega_{t}} \frac{\partial f(x, \tau)}{\partial t} \frac{\partial \bar{\psi}^{N}(x, \tau)}{\partial t} d x d \tau, \quad t \in[0, T]
\end{aligned}
$$

Subtracting the complex conjugate from this equality, we get

$$
\begin{aligned}
\int_{\Omega_{t}} \frac{\partial}{\partial t}\left|\frac{\partial \psi^{N}}{\partial t}\right|^{2} d x d \tau+\int_{\Omega_{t}} \sum_{j=1}^{2} \frac{\partial}{\partial x_{j}}\left(a_{1 j}(x)\left|\frac{\partial \psi^{N}}{\partial t}\right|^{2}\right) d x d \tau \\
+\int_{\Omega_{z}} a_{2}\left(\frac{\partial}{\partial t}\left(\left|\psi^{N}\right|^{2} \psi^{N}\right) \frac{\partial \bar{\psi}^{N}}{\partial t}+\frac{\partial}{\partial t}\left(\left|\psi^{N}\right|^{2} \bar{\psi}^{N}\right) \frac{\partial \psi^{N}}{\partial t}\right) d x d \tau \\
=-\int_{\Omega_{t}} \sum_{j=1}^{2} a_{1 j}(x)\left|\frac{\partial \psi^{N}}{\partial t}\right|^{2} d x d \tau \\
+2 \int_{\Omega_{t}} \operatorname{Im}\left(\frac{\partial f}{\partial t} \frac{\partial \bar{\psi}^{N}}{\partial t}\right) d x d \tau, \quad t \in[0, T]
\end{aligned}
$$

It is clear that the equality

$$
\begin{aligned}
\frac{\partial}{\partial t}\left(\left|\psi^{N}\right|^{2} \psi^{N}\right) \frac{\partial \bar{\psi}^{N}}{\partial t} & +\frac{\partial}{\partial t}\left(\left|\psi^{N}\right|^{2} \bar{\psi}^{N}\right) \frac{\partial \psi^{N}}{\partial t} \\
& =4\left|\psi^{N}\right|^{2}\left|\frac{\partial \psi^{N}}{\partial t}\right|^{2}+2 \operatorname{Re}\left[\left(\psi^{N}\right)^{2}\left(\frac{\partial \bar{\psi}^{N}}{\partial t}\right)\right]
\end{aligned}
$$

holds true. From the other hand, using equality (2.17) and the condition $\left.u_{k}\right|_{\Gamma}=$ $0, k=1,2, \ldots$, we can write

$$
\left.\frac{\partial \psi^{N}}{\partial t}\right|_{S}=0, \quad N=1,2, \ldots
$$

Using (2.28), (2.29), the Cauchy-Bunyakovsky-Schwartz inequality and estimate (2.25), it can be obtained from (2.27) that the inequality

$$
\begin{aligned}
\left\|\frac{\partial \psi^{N}(\cdot, t)}{\partial t}\right\|_{L_{2}(D)}^{2} & +2 a_{2} \int_{\Omega_{t}}\left|\psi^{N}\right|^{2}\left|\frac{\partial \psi^{N}}{\partial t}\right|^{2} d x d \tau \\
& \leq\left\|\frac{\partial \psi^{N}(\cdot, 0)}{\partial t}\right\|_{L_{2}(D)}^{2}+c_{3}\left(\|\phi\|_{L_{2}(D)}^{2}+\|f\|_{L_{2}(\Omega)}^{2}\right) \\
& +\left(2 \mu_{2}+1\right) \int_{0}^{t}\left\|\frac{\partial \psi^{N}(\cdot, t)}{\partial t}\right\|_{L_{2}(D)}^{2} d x d \tau, \quad t \in[0, T]
\end{aligned}
$$

holds true.

To estimate the first term of the right-hand side of this inequality, we use system (2.18) and establish the inequality

$$
\left\|\frac{\partial \psi^{N}(\cdot, 0)}{\partial t}\right\|_{L_{2}(D)}^{2} \leq 5\left\|L \psi^{N}(\cdot, 0)\right\|_{L_{2}(D)}^{2}+5 a_{2}^{2}\left\|\psi^{N}(\cdot, 0)\right\|_{L_{6}(D)}^{6}
$$




$$
+5\|f(\cdot, 0)\|_{L_{2}(D)}^{2}+20 \mu_{1}^{2}\left\|\nabla \psi^{N}(\cdot, 0)\right\|^{2}+5 b_{0}^{2}\left\|\psi^{N}(\cdot, 0)\right\|_{L_{2}(D)}^{2} .
$$

Using the Gagliardo-Nirenberg inequality (see [16, p. 79]), for $n=2$ we have

$$
\left\|\psi^{N}(\cdot, t)\right\|_{L_{6}(D)} \leq \beta\left\|\nabla \psi^{N}(\cdot, t)\right\|_{L_{2}(D)}^{\frac{2}{3}}\left\|\psi^{N}(\cdot, t)\right\|_{L_{2}(D)}^{\frac{1}{3}},
$$

where $\beta>0$ is some constant. With the help of formula (2.17), from this we get

$$
\left\|\varphi^{N}\right\|_{L_{6}(D)} \leq \beta\left\|\nabla \varphi^{N}\right\|_{L_{2}(D)}^{\frac{2}{3}}\left\|\varphi^{N}\right\|_{L_{2}(D)}^{\frac{1}{3}} .
$$

Since $f \in W_{2}^{0,1}(\Omega)$, it is easy to set

$$
\|f(\cdot, t)\|_{L_{2}(D)}^{2} \leq c_{4}\|f\|_{W_{2}^{0,1}(\Omega)}, \quad t \in[0, T] .
$$

It is clear that

$$
L \psi^{N}(x, 0)=\sum_{k=1}^{N} c_{k}^{N}(0) u_{k}(x)=\sum_{k=1}^{N}\left(L \varphi, u_{k}\right)_{L_{2}(D)} u_{k}(x) .
$$

Then we get

$$
\left\|L \psi^{N}(\cdot, 0)\right\|_{L_{2}(D)}^{2}=\sum_{k=1}^{N}\left|\left(L \varphi, u_{k}\right)_{L_{2}(D)} u_{k}(x)\right|^{2} \leq\|L \varphi\|_{l_{2}(D)}^{2} .
$$

It follows from the last inequality, the condition $\varphi \in \stackrel{\circ}{W_{2}^{2}}(D)$ and the conditions set on the coefficients of equation (2.1) that we can get the estimate

$$
\left\|L \psi^{N}(\cdot, 0)\right\|_{L_{2}(D)}^{2} \leq c_{5}\|\phi\|_{W_{2}^{2}(D)}^{2} .
$$

In a similar way, we obtain

$$
\left\|\nabla \psi^{N}(\cdot, 0)\right\|_{L_{2}(D)}^{2} \leq c_{6}\|\phi\|_{W_{2}^{1}(D)}^{2} .
$$

Considering inequalities (2.32)-(2.37) and (2.31), we have

$$
\left\|\frac{\partial \psi^{N}(\cdot, 0)}{\partial t}\right\|_{L_{2}(D)}^{2} \leq c_{7}\left(\|\phi\|_{W_{2}^{2}(D)}^{2}+\|f\|_{W_{2}^{0,1}(\Omega)}^{2}+\|\phi\|_{W_{2}^{1}(D)}^{6}\right) .
$$

Consideration of (2.30) gives

$$
\begin{aligned}
\left\|\frac{\partial \psi^{N}(\cdot, t)}{\partial t}\right\|_{L_{2}(D)}^{2} & +2 a_{2} \int_{\Omega_{t}}\left|\psi^{N}\right|^{2}\left|\frac{\partial \psi^{N}}{\partial t}\right|^{2} d x d \tau \\
& \leq c_{8}\left(\|\phi\|_{W_{2}^{2}(D)}^{2}+\|f\|_{W_{2}^{0,1}(\Omega)}^{2}+\|\phi\|_{W_{2}^{1}(D)}^{6}\right) \\
& +\left(2 \mu_{2}+1\right) \int_{0}^{t}\left\|\frac{\partial \psi^{N}(\cdot, t)}{\partial t}\right\|_{L_{2}(D)}^{2} d x d \tau, \quad t \in[0, T] .
\end{aligned}
$$


Using Gronwall's lemma, one can derive from (2.39):

$$
\begin{aligned}
\left\|\frac{\partial \psi^{N}(\cdot, t)}{\partial t}\right\|_{L_{2}(D)}^{2} & +2 a_{2} \int_{\Omega_{t}}\left|\psi^{N}\right|^{2}\left|\frac{\partial \psi^{N}}{\partial t}\right|^{2} d x d \tau \\
& \leq c_{9}\left(\|\phi\|_{W_{2}^{2}(D)}^{2}+\|f\|_{W_{2}^{0,1}(\Omega)}^{2}+\|\phi\|_{W_{2}^{1}(D)}^{6}\right), t \in[0, T] .
\end{aligned}
$$

To estimate $\nabla \psi^{N}(x, t)$ in the $L_{2}(D)$ norm for any $t \in[0, T]$, we multiply each $k$-th equation of system (2.18) by $\frac{d \bar{c}_{k}^{N}(t)}{d t}$ and take a sum of all obtained equalities over $k=1$ up to $k=N$. Then, integrating the obtained equation on the interval $(0, t)$, we get

$$
\begin{aligned}
& \int_{\Omega_{t}}\left(i\left|\frac{\partial \psi^{N}}{\partial t}\right|^{2}-a_{0} \nabla \psi^{N} \frac{\partial}{\partial t}\left(\nabla \bar{\psi}^{N}\right)+i a_{1}(x) \nabla \psi^{N} \frac{\partial \bar{\psi}^{N}}{\partial t}\right. \\
& \left.\quad+v(x) \psi^{N} \frac{\partial \bar{\psi}^{N}}{\partial t}+i a_{2}\left|\psi^{N}\right|^{2} \psi^{N} \frac{\partial \bar{\psi}^{N}}{\partial t}\right) d x d \tau \int_{\Omega_{t}} f \frac{\partial \bar{\psi}^{N}}{\partial t} d x d \tau, \quad t \in[0, T] .
\end{aligned}
$$

Summing this equality with its complex conjugate and applying to the obtained Cauchy-Bunyakovsky-Schwartz inequality and using then the conditions on the coefficients and estimates (2.25), (2.40), one can easily get the inequality

$$
\begin{aligned}
\left\|\nabla \psi^{N}(\cdot, t)\right\|_{L_{2}(D)}^{2} & \leq\left\|\nabla \psi^{N}(\cdot, 0)\right\|_{L_{2}(D)}^{2} \\
& +c_{10}\left(\|\phi\|_{W_{2}^{2}(D)}^{2}+\|f\|_{W_{2}^{0,1}(\Omega)}^{2}+\|\phi\|_{W_{2}^{1}(D)}^{6}\right), \quad t \in[0, T] .
\end{aligned}
$$

Here the constant $c_{10}>0$ does not depend on $N$. The last inequality and (2.37) give the estimate

$$
\left\|\nabla \psi^{N}(\cdot, t)\right\|_{L_{2}(D)}^{2} \leq c_{11}\left(\|\phi\|_{W_{2}^{2}(D)}^{2}+\|f\|_{W_{2}^{0,1}(\Omega)}^{2}+\|\phi\|_{W_{2}^{1}(D)}^{6}\right), t \in[0, T] .
$$

Here the constant $c_{11}>0$ does not depend on $N$.

Now we estimate $\psi^{N}(x, t)$ in the norm of $\stackrel{\circ}{W}_{2}^{2}(D)$. For this purpose, we multiply each $k$-th equation of system (2.18) by $\lambda_{k} \bar{c}_{k}^{N}(t)$ and sum all obtained equalities over $k=1$ up to $k=N$. Then we get

$$
\begin{array}{r}
\int_{D}\left|L \psi^{N}(x, t)\right|^{2} d x=\int_{D}\left[i \frac{\partial \psi^{N}(x, t)}{\partial t}+i a_{1} \nabla \psi^{N}(x, t)+v(x) \psi^{N}(x, t)\right. \\
\left.+i a_{2}\left|\psi^{N}(x, t)\right|^{2} \psi^{N}(x, t)-f(x, t)\right] L \bar{\psi}^{N}(x, t) d x, \quad t \in[0, T] .
\end{array}
$$

From this equation, using the Cauchy-Bunyakovsky-Schwartz inequality, we obtain the inequality

$$
\left\|L \psi^{N}(\cdot, t)\right\|_{L_{2}(D)}^{2} \leq 5\left\|\frac{\partial \psi^{N}(\cdot, t)}{\partial t}\right\|_{L_{2}(D)}^{2}+5 \mu_{2}^{2}\left\|\nabla \psi^{N}(\cdot, t)\right\|_{L_{2}(D)}^{2}
$$




$$
+5 a_{2}^{2}\left\|\psi^{N}(\cdot, t)\right\|_{L_{6}(D)}^{6}+5 b_{0}^{2}\left\|\psi^{N}(\cdot, t)\right\|_{L_{2}(D)}^{2}+5\|f(\cdot, t)\|_{L_{2}(D)}^{2}, t \in[0, T] .
$$

With the help of inequalities (2.32), (2.34) and estimates (2.25), (2.40), (2.41), from (2.43) we get

$$
\left\|L \psi^{N}(\cdot, t)\right\|_{L_{2}(D)}^{2} \leq c_{12}\left(\|\phi\|_{W_{2}^{2}(D)}^{2}+\|f\|_{W_{2}^{0,1}(\Omega)}^{2}+\|\phi\|_{W_{2}^{1}(D)}^{6}\right), t \in[0, T] .
$$

Here the constant $c_{12}>0$ does not depend on $N$. By the definition of the operator $L$, we have

$$
\begin{aligned}
\left\|L \psi^{N}(\cdot, t)\right\|_{L_{2}(D)} & =\left\|-a_{0} \Delta \psi^{N}(\cdot, t)+a(\cdot) \psi^{N}(\cdot, t)\right\|_{L_{2}(D)} \\
& \geq a_{0}\left\|\Delta \psi^{N}(\cdot, t)\right\|_{L_{2}(D)}-\mu_{0}\left\|\psi^{N}(\cdot, t)\right\|_{L_{2}(D)}
\end{aligned}
$$

This implies

$$
\left\|\Delta \psi^{N}(\cdot, t)\right\|_{L_{2}(D)} \leq \frac{1}{a_{0}}\left\|L \psi^{N}(\cdot, t)\right\|_{L_{2}(D)}+\frac{\mu_{0}}{a_{0}}\left\|\psi^{N}(\cdot, t)\right\|_{L_{2}(D)} .
$$

Substituting (2.25) and (2.44) in this inequality, we get the validity of

$$
\left\|\Delta \psi^{N}(\cdot, t)\right\|_{L_{2}(D)}^{2} \leq c_{13}\left(\|\phi\|_{W_{2}^{2}(D)}^{2}+\|f\|_{W_{2}^{0,1}(\Omega)}^{2}+\|\phi\|_{W_{2}^{2}(D)}^{6}\right) .
$$

Here the constant $c_{13}>0$ does not depend on $N$. Using the well-known inequality (see $[11$, p. 124]), for the convex domain $D$ we obtain

$$
\left\|\psi^{N}(\cdot, t)\right\|_{W_{2}^{2}(D)}^{2} \leq c_{14}\left\|\Delta \psi^{N}(\cdot, t)\right\|, t \in[0, T] .
$$

Consideration of (2.45) and (2.46) gives

$$
\left\|\psi^{N}(\cdot, t)\right\|_{W_{2}^{2}(D)}^{2} \leq c_{15}\left(\|\phi\|_{W_{2}^{2}(D)}^{2}+\|f\|_{W_{2}^{0,1}(\Omega)}^{2}+\|\phi\|_{W_{2}^{1}(D)}^{6}\right), t \in[0, T] .
$$

Here the constant $c_{15}>0$ does not depend on $N$. Thus, taking into account estimates (2.40) and (2.47), we finally get

$$
\begin{aligned}
\left\|\psi^{N}(\cdot, t)\right\|_{W_{2}^{2}(D)}^{2} & +\left\|\frac{\partial \psi^{N}(\cdot, t)}{\partial t}\right\|_{L_{2}(D)}^{2} \\
& \leq c_{16}\left(\|\phi\|_{W_{2}^{2}(D)}^{2}+\|f\|_{W_{2}^{0,1}(\Omega)}^{2}+\|\phi\|_{W_{2}^{\prime}(D)}^{6}\right), t \in[0, T],
\end{aligned}
$$

where the constant $c_{16}>0$ does not depend on $N$. Using this estimate and the inequality

$$
\begin{aligned}
\sum_{k=1}^{N}\left|c_{k}^{N}(t)\right|^{2} & +\sum_{k=1}^{N}\left|\frac{d c_{k}^{N}(t)}{d t}\right|^{2} \\
& \leq\left\|\psi^{N}(\cdot, t)\right\|_{W_{2}^{2}(D)}^{2}+\left\|\frac{\partial \psi^{N}(\cdot, t)}{\partial t}\right\|_{L_{2}(D)}^{2}, \quad t \in[0, T],
\end{aligned}
$$

denoting $c_{1}=c_{16}$, we come to the statement of the lemma. Lemma 2.3 is proved. 
Now we continue the proof of the theorem. Let us consider the functions $l_{N, k}(t)=\left(\psi^{N}(\cdot, t), u_{k}\right)_{L_{2}(D)}, N, k=1,2, \ldots$ It follows from $(2.20)$ and orthogonality of the functions $u_{k}=u_{k}(x), k=1,2, \ldots$, that the families of functions $l_{N, k}(t), N, k=1,2, \ldots$, and their derivatives $\frac{d l_{N, k}(t)}{d t}, N, k=1,2, \ldots$, are uniformly bounded on the interval $[0, T]$,

$$
\left|l_{N, k}(t)\right| \leq c_{17}, \quad\left|\frac{d l_{N, k}(t)}{d t}\right| \leq c_{18}, \quad N, k=1,2, \ldots, t \in[0, T] .
$$

Let us show that for the fixed $k$ and any $N \geq k$, the functions $l_{N, k}(t), N, k=$ $1,2, \ldots$, are equicontinuous on the interval $[0, T]$. Indeed, integrating the $k$-th equation from (2.18) on the interval $[t, t+\Delta t]$, we get

$$
\begin{aligned}
\left|l_{N, k}(t+\Delta t)-l_{N, k}(t)\right| & \leq \int_{t}^{t+\Delta t}\left|\int_{D} a_{0} \Delta \psi^{N}(x, \tau) u_{k}(x) d x\right| d \tau \\
& +\int_{t}^{t+\Delta t}\left|\int_{D} i a_{1}(x) \nabla \psi^{N}(x, \tau) u_{k}(x) d x\right| d \tau \\
& +\int_{t}^{t+\Delta t}\left|\int_{D} a(x) \psi^{N}(x, \tau) u_{k}(x) d x\right| d \tau \\
& +\int_{t}^{t+\Delta t}\left|\int_{D} v(x) \psi^{N}(x, \tau) u_{k}(x) d x\right| d \tau \\
& +\left.\int_{t}^{t+\Delta t}\left|\int_{D} i a_{2}\right| \psi^{N}(x, \tau)\right|^{2} \psi^{N}(x, \tau) u_{k}(x) d x \mid d \tau \\
& +\int_{t}^{t+\Delta t}\left|\int_{D} f(x, \tau) u_{k}(x) d x\right| d \tau
\end{aligned}
$$

Together with the Cauchy-Bunyakovsky-Schwartz inequality this gives the inequality

$$
\begin{aligned}
\left|l_{N, k}(t+\Delta t)-l_{N, k}(t)\right| & \leq a_{0} \int_{t}^{t+\Delta t}\left\|\Delta \psi^{N}(\cdot, \tau)\right\|_{L_{2}(D)}\left\|u_{k}\right\|_{L_{2}(D)} d \tau \\
& +\sqrt{2} \mu_{1} \int_{t}^{t+\Delta t}\left\|\nabla \psi^{N}(\cdot, \tau)\right\|_{L_{2}(D)}\left\|u_{k}\right\|_{L_{2}(D)} d \tau \\
& +\left(\mu_{0}+b_{0}\right) \int_{t}^{t+\Delta t}\left\|\psi^{N}(\cdot, \tau)\right\|_{L_{2}(D)}\left\|u_{k}\right\|_{L_{2}(D)} d \tau \\
& +a_{2} \int_{t}^{t+\Delta t}\left\|\psi^{N}(\cdot, \tau)\right\|_{L_{6}(D)}^{3}\left\|u_{k}\right\|_{L_{2}(D)} d \tau \\
& +\int_{t}^{t+\Delta t}\|f(\cdot, \tau)\|_{L_{2}(D)}\left\|u_{k}\right\|_{L_{2}(D)} d \tau .
\end{aligned}
$$

Therefore, taking into account (2.32), estimates (2.20), (2.45) and assumption (2.16), we get the relation

$$
\left|l_{N, k}(t+\Delta t)-l_{N, k}(t)\right| \leq c_{19} d_{k} \Delta t, \quad N, k=1,2, \ldots,
$$


where the constant $c_{19}>0$ does not depend on $N, k, t$.

Performing the integration by parts in the second and third terms of the left-hand side of equations (2.18) and differentiating the obtained relations with respect to $t$, and then integrating on the interval $[t, t+\Delta t]$, one can get

$$
\begin{aligned}
\mid \frac{d l_{N, k}(t+\Delta t)}{d t} & -\frac{d l_{N, k}(t)}{d t}\left|\leq \int_{t}^{t+\Delta t}\right| \int_{D} a_{0} \frac{\partial \psi^{N}(x, \tau)}{\partial \tau} \Delta u_{k}(x) d x \mid d \tau \\
& +\int_{t}^{t+\Delta t}\left|\int_{D} i \frac{\partial \psi^{N}(x, \tau)}{\partial \tau} \nabla\left(a_{1}(x) u_{k}(x)\right) d x\right| d \tau \\
& +\int_{t}^{t+\Delta t}\left|\int_{D} a(x) \frac{\partial \psi^{N}(x, \tau)}{\partial \tau} u_{k}(x) d x\right| d \tau \\
& +\int_{t}^{t+\Delta t}\left|\int_{D} v(x) \frac{\partial \psi^{N}(x, \tau)}{\partial \tau} u_{k}(x) d x\right| d \tau \\
& +\int_{t}^{t+\Delta t}\left|\int_{D} i a_{2} \frac{\partial}{\partial \tau}\left(\left|\psi^{N}(x, \tau)\right|^{2} \psi^{N}(x, \tau)\right) u_{k}(x) d x\right| d \tau \\
& +\int_{t}^{t+\Delta t}\left|\int_{D} \frac{\partial f(x, \tau)}{\partial \tau} u_{k}(x) d x\right| d \tau .
\end{aligned}
$$

From this, by virtue of Cauchy-Bunyakovsky-Schwartz inequality, we get

$$
\begin{aligned}
\mid \frac{d l_{N, k}(t+\Delta t)}{d t} & -\frac{d l_{N, k}(t)}{d t} \mid \leq a_{0} \int_{t}^{t+\Delta t}\left\|\frac{\partial \psi(\cdot, \tau)}{\partial \tau}\right\|_{L_{2}(D)} d \tau\left\|\Delta u_{k}\right\|_{L_{2}(D)} \\
& +\sqrt{2} \mu_{1} \int_{t}^{t+\Delta t}\left\|\frac{\partial \psi(\cdot, \tau)}{\partial \tau}\right\|_{L_{2}(D)} d \tau\left\|\nabla u_{k}\right\|_{L_{2}(D)} \\
& +\left(\mu_{0}+b_{0}+\sqrt{2} \mu_{2}\right) \int_{t}^{t+\Delta t}\left\|\frac{\partial \psi(\cdot, \tau)}{\partial \tau}\right\|_{L_{2}(D)} d \tau\left\|u_{k}\right\|_{L_{2}(D)} \\
& +3 a_{2} \int_{t}^{t+\Delta t} \int_{D}\left|\frac{\partial \psi^{N}(x, \tau)}{\partial \tau}\right||\psi(x, \tau)|^{2}\left|u_{k}(x)\right| d x d \tau \\
& +\int_{t}^{t+\Delta t}\left\|\frac{\partial f(\cdot, \tau)}{\partial \tau}\right\|_{L_{2}(D)} d \tau\left\|u_{k}\right\|_{L_{2}(D)} \cdot
\end{aligned}
$$

Now, let us estimate the fourth term of the right-hand side of this inequality.

Then, by virtue of the Cauchy-Bunyakovsky-Schwartz inequality, we get

$$
\begin{aligned}
3 a_{2} \int_{t}^{t+\Delta t} \int_{D}\left|\frac{\partial \psi^{N}(x, \tau)}{\partial \tau}\right| & \left|\psi^{N}(x, \tau)\right|^{2}\left|u_{k}(x)\right| d x d \tau \\
\leq & 3 a_{2} \int_{t}^{t+\Delta t}\left(\int_{D}\left|\frac{\partial \psi^{N}(x, \tau)}{\partial \tau}\right|^{2}\left|\psi^{N}(x, \tau)\right|^{2} d x\right)^{\frac{1}{2}} \\
& \times\left(\int_{D}\left|\psi^{N}(x, \tau)\right|^{2}\left|u_{k}(x)\right|^{2} d x\right)^{\frac{1}{2}} d \tau
\end{aligned}
$$

If we apply the Cauchy-Bunyakovsky-Schwartz inequality to the second multiplier in the integrant in the right-hand side of this inequality, then we get 


$$
\begin{aligned}
\left(\int_{D}\left|\psi^{N}(x, \tau)\right|^{2}\left|u_{k}(x)\right|^{2}\right. & d x)^{\frac{1}{2}} \\
& \leq\left(\int_{D}\left|\psi^{N}(x, \tau)\right|^{4} d x\right)^{\frac{1}{4}}\left(\int_{D}\left|u_{k}(x)\right|^{4} d x\right)^{\frac{1}{4}}
\end{aligned}
$$

By virtue of the inequalities from [13, pp. 84 and 88], we have

$$
\begin{aligned}
\left\|u_{k}\right\|_{L_{4}(D)} & \leq c_{20}\left\|\nabla u_{k}\right\|_{L_{2}(D)}, \\
\left\|\psi^{N}(\cdot, \tau)\right\|_{L_{4}(D)} & \leq c_{21}\left\|\psi^{N}(\cdot, \tau)\right\|_{W_{2}^{1}(D)} .
\end{aligned}
$$

Then, taking into account (2.53)-(2.55), from (2.52) we get

$$
\begin{aligned}
3 a_{2} \int_{t}^{t+\Delta t} \int_{D} \mid & \left.\frac{\partial \psi^{N}(x, \tau)}{\partial \tau}|| \psi^{N}(x, \tau)\right|^{2}\left|u_{k}(x)\right| d x d \tau \\
\leq & 3 c_{20} c_{21} a_{2} \int_{t}^{t+\Delta t}\left(\int_{D}\left|\frac{\partial \psi^{N}(x, \tau)}{\partial \tau}\right|^{2}\left|\psi^{N}(x, \tau)\right|^{2} d x\right)^{\frac{1}{2}} \\
& \times\left\|\psi^{N}(\cdot, \tau)\right\|_{W_{2}^{1}(D)} d \tau\left\|\nabla u_{k}\right\|_{L_{2}(D)}
\end{aligned}
$$

Substituting (2.56) into (2.51), with the help of (2.20), (2.40) and assumption (2.16) it is easy to establish the inequality

$$
\left|\frac{d l_{N, k}(t+\Delta t)}{d t}-\frac{d l_{N, k}(t)}{d t}\right| \leq c_{22} d_{k}(\Delta t)^{\frac{1}{2}}, \quad N, k=1,2, \ldots,
$$

where the constant $c_{22}>0$ does not depend on $N, k, t$.

It follows from (2.50) and (2.57) that the families of functions $\left\{l_{N, k}(t)\right\}$, $\left\{\frac{d l_{N, k}(t)}{d t}\right\}, N, k=1,2, \ldots$, are equicontinuous on the interval $[0, T]$ for a fixed $k$ and arbitrary $N \geq k$. Then, by a standard diagonal procedure, we can choose a subsequence $N_{m}, m=1,2, \ldots$, such that the corresponding functions $l_{N_{m}, k}(t)$, $m=1,2, \ldots$ and their derivatives $\frac{d l_{N_{m}, k}(t)}{d t}, m=1,2, \ldots$, converge uniformly on the interval $[0, T]$ to the continuous functions $l_{k}(t), \frac{d l_{k}(t)}{d t}$ for each $k=1,2, \ldots$. The functions $l_{k}(t), k=1,2, \ldots$, and their derivatives define the functions

$$
\psi(x, t)=\sum_{k=1}^{\infty} l_{k}(t) u_{k}(x), \quad \frac{\partial \psi(x, t)}{\partial t}=\sum_{k=1}^{\infty} \frac{d l_{k}(t)}{d t} u_{k}(x) .
$$

Then, as in $[4,8]$, we can state the subsequences $\left\{\psi^{N_{m}}(x, t)\right\},\left\{\frac{\partial \psi^{N_{m}}(x, t)}{\partial t}\right\}$, defined by formulas (2.58), converge weakly in $\stackrel{\circ}{W_{2}^{2}}(D), L_{2}(D)$ to the functions $\psi(x, t), \frac{\partial \psi(x, t)}{\partial t}$, respectively, uniformly with respect to $t \in[0, T]$. The limit function $\psi(x, t)$ belongs to the space $B_{0}$.

Now we show that the limit function $\psi(x, t)$ is a solution of problem (2.1), (2.2) in the sense of Definition 2.1. For this purpose, we first prove that this 
function satisfies equation (2.1) for almost all $x \in D$ and arbitrary $t \in[0, T]$. We set $N=N_{m}$ and multiply the $k$-th equation from (2.18) by a continuous function $\bar{\eta}_{k}(t)$ and sum up the obtained equations with respect to $k$ from $k=1$ to $N^{\prime} \leq$ $N_{m}$. Then we get

$$
\begin{aligned}
\int_{D}\left(i \frac{\partial \psi^{N_{m}}(x, t)}{\partial t}\right. & -a_{0} \Delta \psi^{N_{m}}(x, t)+i a_{1}(x) \nabla \psi^{N_{m}}(x, t)-a(x) \psi^{N_{m}}(x, t) \\
& +v(x) \psi^{N_{m}}(x, t)+i a_{2}\left|\psi^{N_{m}}(x, t)\right|^{2} \psi^{N_{m}}(x, t) \\
& -f(x, t)) \bar{\eta}^{N^{\prime}}(x, t) d x=0, \quad t \in[0, T]
\end{aligned}
$$

for any function $\bar{\eta}_{k}^{N^{\prime}}(x, t)=\sum_{k=1}^{N^{\prime}} \bar{\eta}_{k}(t) u_{k}(x), N^{\prime} \leq N_{m}$.

The sequence $\left\{\psi^{N_{m}}(x, t)\right\}$ converges uniformly to the function $\psi=\psi(x, t)$ as $m \rightarrow \infty$, and the space $\stackrel{\circ}{W_{2}^{2}}(D)$ is compact embedded into $L_{2}(D)$ (see $[15,16,19]$ ). Therefore, there exists a subsequence of $\left\{\psi^{N_{m}}(x, t)\right\}$ which converges strongly in $L_{2}(D)$ to the function $\psi=\psi(x, t)$ as $m \rightarrow \infty$, i.e.,

$$
\left\|\psi^{N_{m}}(\cdot, t)-\psi(\cdot, t)\right\|_{L_{2}(D)} \rightarrow 0
$$

uniformly with respect to $t \in[0, T]$ as $m \rightarrow \infty$. Consequently, there exists a subsequence of $\left\{\psi^{N_{m}}(x, t)\right\}$ which converges to the function $\psi=\psi(x, t)$ almost everywhere in $D$. For the sake of simplicity, this subsequence is denoted again by $\left\{\psi^{N_{m}}(x, t)\right\}$. Then we can write

$$
\psi^{N_{m}}(x, t) \rightarrow \psi(x, t) \quad \text { almost everywhere in } D
$$

uniformly with respect to $t \in[0, T]$ as $m \rightarrow \infty$. Besides, due to uniform estimate (2.20) and inequality (2.32), for $N=N_{m}$, the inequality

$$
\left\|\left|\psi^{N_{m}}(\cdot, t)\right|^{2} \psi(\cdot, t)\right\|_{L_{2}(D)} \leq\left\|\psi^{N_{m}}(\cdot, t)\right\|_{L_{2}(D)}^{3}
$$

holds true. From the known lemma (see [13, pp. 530-531]), we obtain that $\left\{\left|\psi^{N_{m}}(x, t)\right|^{2} \psi^{N_{m}}(x, t)\right\}$ converges weakly in $L_{2}(D)$ to the function $|\psi(x, t)|^{2} \psi(x, t)$ uniformly with respect to $t \in[0, T]$ as $m \rightarrow \infty$, i.e.,

$$
\begin{aligned}
\int_{D}\left|\psi^{N_{m}}(x, t)\right|^{2} \psi^{N_{m}}(x, t) \bar{\eta}^{N^{\prime}}(x, t) d x & \\
& \rightarrow \int_{D}|\psi(x, t)|^{2} \psi(x, t) \bar{\eta}^{N^{\prime}}(x, t) d x \quad \text { as } m \rightarrow \infty t \in[0, T]
\end{aligned}
$$

for any continuous on the interval $[0, T]$ in the $L_{2}(D)$ norm function $\bar{\eta}_{k}^{N^{\prime}}(x, t)=$ $\sum_{k=1}^{N^{\prime}} \bar{\eta}_{k}(t) u_{k}(x), N^{\prime} \leq N_{m}$. Using this limit relation and the convergence of the subsequence $\left\{\psi^{N_{m}}(x, t)\right\}$ to the function $\psi(x, t)$, passing to limit as $m \rightarrow \infty$, in (2.59), we get

$$
\int_{D}\left(i \frac{\partial \psi(x, t)}{\partial t}-a_{0} \Delta \psi(x, t)+i a_{1}(x) \nabla \psi(x, t)-a(x) \psi(x, t)+v(x) \psi(x, t)\right.
$$




$$
\left.+i a_{2}|\psi(x, t)|^{2} \psi(x, t)-f(x, t)\right) \bar{\eta}^{N^{\prime}}(x, t) d x=0, \quad t \in[0, T]
$$

for any function $\bar{\eta}_{k}^{N^{\prime}}(x, t)=\sum_{k=1}^{N^{\prime}} \bar{\eta}_{k}(t) u_{k}(x), N^{\prime} \leq N_{m}$. Since all functions of the form $\bar{\eta}_{k}^{N^{\prime}}(x, t)=\sum_{k=1}^{N^{\prime}} \bar{\eta}_{k}(t) u_{k}(x)$ are dense in $C^{0}\left([0, T], L_{2}(D)\right)$, we obtain immediately from identity (2.63) that the limit function $\psi(x, t)$ satisfies equation (2.1) for any $t \in[0, T]$ and for almost all $x \in D$. The fulfillment of the initial and boundary conditions (2.2) for the limit function $\psi(x, t)$ follows from the limit relation (2.60) for $t=0$ and the fact that the space $B_{0}$ is compactly embedded into $L_{2}(S)$.

Thus, we have proved that the limit function $\psi(x, t)$ is a solution of the initial-boundary problem (2.1), (2.2), and this solution belongs to the space $B_{0}$ and satisfies (2.7), which follows immediately from (2.20) after passing to the lower limit over the weakly convergent subsequence $\left\{\psi^{N_{m}}(x, t)\right\}$ from $B_{0}$ to the function $\psi(x, t)$.

Now, continuing the proof of the theorem, we prove the uniqueness of the solution of the initial-boundary value problem (2.1), (2.2). Let $\psi(x, t)$ and $\Phi(x, t)$ be two arbitrary solutions for problem (2.1), (2.2). Let $w(x, t)=\psi(x, t)-\Phi(x, t)$. Then it is clear from condition $(2.1),(2.2)$ that $w=w(x, t)$ can be a solution of the following initial-boundary problem:

$$
\begin{aligned}
& i \frac{\partial w}{\partial t}+a_{0} \Delta w+i a_{1}(x) \nabla w-a(x) w+v(x) w \\
&+i a_{2}\left(|\psi|^{2}+|\Phi|^{2}\right) w+i a_{1} \psi \Phi \bar{w}=0, \quad(x, t) \in \Omega \\
& w(x, 0)=0, \quad x \in D,\left.\quad w\right|_{S}=0 .
\end{aligned}
$$

To establish the estimate for the solution of this problem, we multiply (2.64) by the function $\bar{w}(x, t)$ and integrate on the domain $\Omega_{t}$. Using the boundary condition from (2.65) and integrating by parts, we get

$$
\begin{aligned}
\int_{\Omega_{t}}\left(i \frac{\partial w}{\partial t} \bar{w}-\right. & a_{0}|\nabla w|^{2}+i a_{1}(x) \nabla w \bar{w}-a(x)|w|^{2}+v(x)|w|^{2} \\
& \left.+i a_{2}\left(|\psi|^{2}+|\Phi|^{2}\right)|w|^{2}+i a_{1} \psi \Phi(\bar{w})^{2}\right) d x d \tau=0, \quad t \in[0, T] .
\end{aligned}
$$

Subtracting from this equality its complex conjugate and using boundary condition (2.65), we obtain

$$
\begin{aligned}
\int_{\Omega_{t}} i\left(\frac{\partial w}{\partial \tau} \bar{w}\right. & \left.+\frac{\partial \bar{w}}{\partial \tau} w\right) d x d \tau+i 2 a_{2} \int_{\Omega_{t}}\left(|\psi|^{2}+|\Phi|^{2}\right)|w|^{2} d x d \tau \\
& =-i 2 a_{2} \int_{\Omega_{t}} \operatorname{Im}\left[\psi \Phi(\bar{w})^{2}\right] d x d \tau-i \int_{\Omega_{t}} \sum_{j=1}^{2} \frac{\partial a_{1 j}(x)}{\partial x_{j}}|w|^{2} d x d \tau
\end{aligned}
$$

for any $t \in[0, T]$. Together with (2.65) this gives

$$
\|w(\cdot, t)\|_{L_{2}(D)}^{2}+2 a_{2} \int_{\Omega_{t}}\left(|\psi|^{2}+|\Phi|^{2}\right)|w|^{2} d x d \tau
$$




$$
+2 a_{2} \int_{\Omega_{t}}|\psi||\Phi||w|^{2} d x d \tau+2 \mu_{2} \int_{\Omega_{t}}|w|^{2} d x d \tau
$$

for any $t \in[0, T]$. Application of the inequality $2|\psi||\Phi| \leq|\psi|^{2}+|\Phi|^{2}$ implies

$$
\|w(\cdot, t)\|_{L_{2}(D)}^{2}+a_{2} \int_{\Omega_{t}}\left(|\psi|^{2}+|\Phi|^{2}\right)|w|^{2} d x d \tau \leq 2 \mu_{2} \int_{0}^{t}\|w(\cdot, \tau)\|_{L_{2}(D)}^{2} d \tau
$$

for any $t \in[0, T]$. With the help of Gronwall's lemma, we get the relation

$$
\|w(\cdot, t)\|_{L_{2}(D)}^{2}=0, \quad t \in[0, T],
$$

which proves the validity of

$$
w(x, t)=0, \quad x \in D, t \in[0, T] .
$$

The uniqueness of the solution of the initial-boundary problem (2.1), (2.2) follows immediately. Theorem 2.2 is proved.

Remark 2.4. A similar result can be established when the set $D$ belongs to $R^{3}$.

\section{The existence and uniqueness of a solution of the second initial-boundary value problem}

Consider the initial-boundary problem on determining the function $\psi=$ $\psi(x, t)$ in the domain $\Omega$ subject to

$$
\begin{aligned}
& i \frac{\partial \psi}{\partial t}+a_{0} \Delta \psi+i a_{1}(x) \nabla \psi-a(x) \psi \\
&+v(x) \psi+i a_{2}|\psi|^{2} \psi=f, \quad(x, t) \in \Omega, \\
& \psi(x, 0)=\phi(x), \quad x \in D,\left.\quad \frac{\partial \psi}{\partial \nu}\right|_{S}=0,
\end{aligned}
$$

where $i=\sqrt{-1} ; a_{0}>0, a_{2}>0$ are given numbers; $\nu$ is an outward normal to the boundary $\Gamma ; \Delta=\frac{\partial^{2}}{\partial x_{1}^{2}}+\frac{\partial^{2}}{\partial x_{2}^{2}}$ is the Laplace operator; $\nabla=\left(\frac{\partial}{\partial x_{1}}, \frac{\partial}{\partial x_{2}}\right), a(x), v(x)$ are measurable bounded functions satisfying the conditions

$$
\begin{gathered}
\mu_{0} \leq a(x) \leq \mu_{1}, \quad x \in D, \mu_{0}, \mu_{1}=\text { const }>0 \\
|v(x)| \leq b_{0}, \quad x \in D, \quad b_{0}=\text { const }>0
\end{gathered}
$$

$a_{1}(x)=\left(a_{11}(x), a_{12}(x)\right)$ is a given vector-function whose components satisfy the conditions

$$
\left|a_{1 j}(x)\right| \leq \mu_{2},\left|\frac{\partial a_{1 j}(x)}{\partial x_{k}}\right| \leq \mu_{3}, \quad x \in D, j, k=1,2, \mu_{2}, \mu_{3}=\text { const }>0 ;
$$

$\phi(x), f(x, t)$ are complex valued functions satisfying the conditions

$$
\phi \in W_{2}^{2}(D),\left.\quad \frac{\partial \phi}{\partial \nu}\right|_{\Gamma}=0, \quad f \in W_{2}^{0,1}(\Omega) .
$$


Definition 3.1. The function $\psi=\psi(x, t)$ from the space

$$
B_{1} \equiv C^{0}\left([0, T], W_{2}^{2}(D)\right) \bigcap C^{1}\left([0, T], L_{2}(D)\right)
$$

is called a generalized solution of (3.1), (3.2) if it satisfies equation (3.1) for almost all $x \in D$ and any $t \in[0, T]$, and initial and boundary conditions (3.2) for almost all $x \in D$ and for almost all $(\xi, t) \in S$, respectively.

Theorem 3.2. Let the functions a $(x), v(x), a_{1}(x), \varphi(x), f(x, t)$ satisfy conditions (3.3)-(3.6). Then the initial-boundary problem (3.1), (3.2) has the only solution from the space $B_{1}$, and for this solution the estimate

$$
\begin{aligned}
\|\psi(\cdot, t)\|_{W_{2}^{2}(D)} & +\left\|\frac{\partial \psi(\cdot, t)}{\partial t}\right\|_{L_{2}(D)} \\
& \leq c_{23}\left(\|\phi\|_{W_{2}^{2}(D)}+\|f\|_{W_{2}^{0,1}(\Omega)}+\|\phi\|_{W_{2}^{1}(D)}^{3}\right), \quad t \in[0, T],
\end{aligned}
$$

where the constant $c_{23}>0$ does not depend on $\phi, f$, and $t$, is valid.

This theorem can be proved by using Galerkin's approximations in the same way as Theorem 1 . In this case, as a fundamental in $W_{2}^{2}(D)$ system of functions we take an orthonormal in $L_{2}(D)$ and orthogonal in $W_{2}^{2}(D)$ system $u_{k}=u_{k}(x)$, $k=1,2, \ldots$, of eigenfunction of the spectral problem

$$
L X(x)=\lambda X(z), \quad x \in D,\left.\quad \frac{\partial X}{\partial \nu}\right|_{\Gamma}=0
$$

at $\lambda=\lambda_{k}, k=1,2, \ldots$, where the operator $L$ is defined as

$$
L=-a_{0} \Delta+a(x)
$$

with the Neumann boundary conditions.

Remark 3.3. A similar result is valid when the set $D$ lies in $R^{3}$.

\section{References}

[1] G.D. Akbaba, The Optimal Control Problem with the Lions Functional for the Schrödinger Equation Including Virtual Coefficient Gradient, Master's thesis, Kars (Turkey), 2011 (Turkish).

[2] L. Baudouin, O. Kavian, and J.P. Puel, Regularity for a Schrödinger equation with singular potentials and application to bilinear optimal control, J. Differential Equations 216 (2005), 188-222.

[3] A.G. Butkovskiy and Y.I. Samojlenko, Control of Quantum-Mechanical Processes and Systems. Mathematics and its Applications (Soviet Series), 56, Kluwer Academic Publishers Group, Dordrecht, 1990.

[4] N.S. Ibragimov, The solvability of the initial-boundary value problems for the nonlinear stationary equation of quasi-optics with purely imaginary coefficient in the nonlinear part, News of Baku State University, Ser. Physics and Math. Sciences (2010), No. 3, 72-84. 
[5] A.D. Iskenderov and G.Y. Yagubov, A variational method for solving an inverse problem of determining the quantum mechanical potential, Dokl. Akad. Nauk SSSR 303 (1988), 1044-1048 (Russian); Engl. transl.: Soviet Math. Dokl. 38 (1989), 637641.

[6] A.D. Iskenderov and G.Y. Yagubov, Optimal control of nonlinear quantummechanical systems, Avtomat. i Telemekh. (1989), No. 12, 27-38 (Russian); Engl. transl.: Automat. Remote Control 50 (1989), No. 12, Part 1, 1631-1641 (1990).

[7] A. Iskenderov and G. Yagubov, Optimal control of the unbounded potential in the multidimensional nonlinear nonstationary Schrödinger equation, Bulletin of Lankaran State University, Ser. Natural Sciences (2007), 3-56.

[8] A.D. Iskenderov, G.Y. Yagubov, and M.A. Musayeva, Identification of the Quantum Potentials, Chashyoglu, Baku, 2012 (Azerbaijani).

[9] M. Jahanshahi, S. Ashrafi, and N. Aliev, Boundary layer problem for the system of the first order ordinary differential equations with constant coefficients by general nonlocal boundary conditions, Adv. Math. Models Appl. 2 (2017), 107-116.

[10] O.A. Ladyzhenskaya, Boundary-Value Problems of Mathematical Physics, Nauka, Moscow, 1973 (Russian).

[11] O.A. Ladyzhenskaya, V.A. Solonnikov, and N.N. Ural'tseva, Linear and QuasiLinear Equations of Parabolic Type, Nauka, Moscow, 1967 (Russian); Engl. transl.: Translations of Mathematical Monographs, 23, Amer. Math. Soc., Providence, R.I., 1968.

[12] J.-L. Lions and E. Magenes, Non-Homogeneous Boundary Value Problems and Applications, I, Die Grundlehren der mathematischen Wissenschaften, 181, SpringerVerlag, New York-Heidelberg, 1972.

[13] L.S. Pontryagin, Ordinary Differential Equations, Nauka, Moscow, 1982.

[14] F.P. Vasilyev, Numerical Methods for Solving of the Extremal Problems, Nauka, Moscow, 1980.

[15] M.A. Vorontsov and V.I. Schmalhausen, The Principles of Adaptive Optics, Nauka, Moscow, 1985 (Russian).

[16] G.Y. Yagubov and M.A. Musayeva, On an identification problem for nonlinear Schrödinger equation, Differ. Uravn. 33 (1997), 1691-1698.

[17] G. Yagubov, F. Toyğolu, and M. Subaşı, An optimal control problem for twodimensional Schrödinger equation, Appl. Math. Comput. 218 (2012), 6177-6187.

[18] K. Yajima and G. Zhang, Smoothing property for Schrödinger equations with potential superquadratic at infinity, Comm. Math. Phys. 221 (2001), 573-590.

[19] V.M. Zhuravlev, Nonlinear Waves in Multicomponent Systems Dispersion and Diffusion, Ulyanovsk State University, Ulyanovsk, 2001 (Russian).

Received February 13, 2017, revised January 10, 2018.

G. Yagub,

Kafkas University, Paşaçayırı Campus, Kars, 36040, Turkey,

E-mail: gabilya@mail.ru 
N.S. Ibrahimov,

Baku State University, 23 Academic Zahid Khalilov St., Baku, AZ 1148, Azerbaijan; Lankaran State University, 50 Hazi Aslanov St., Lankaran, AZ 4200, Azerbaijan, E-mail: natiq_ibrahimov@mail.ru

M. Zengin,

Kafkas University, Paşaçayırı Campus, Kars, 36040, Turkey,

E-mail: merveezengin14@gmail.com

\section{Розв'язок початково-крайової задачі для нелінійного рівняння Шредінгера із спеціальним градієнтним членом}

G. Yagub, N.S. Ibrahimov, and M. Zengin

У статті розглядаються початково-крайові задачі для двовимірного нелінійного рівняння Шредінгера із спеціальним градієнтним членом з чисто уявними коефіцієнтами в нелінійній частині, коли коефіцієнти рівняння є вимірними обмеженими функціями. Доведено існування і єдність розв'язків першої і другої початково-крайової задачі майже скрізь.

Ключові слова: рівняння Шредінгера, спеціальний градієнтний член, існування та єдність, перша і друга початково-крайові задачі. 